\title{
CHARACTERIZATION AND MODELING OF PERFORMANCE OF POLYMER COMPOSITES REINFORCED WITH HIGHLY NON-LINEAR CELLULOSIC FIBERS
}

\author{
Liva Rozite $^{1}$, Roberts Joffe ${ }^{1,2}$, Janis Varna ${ }^{1}$, Birgitha Nyström ${ }^{2}$ \\ ${ }^{1}$ Material Science, Luleå University of Technology, S-97187 Luleå, SWEDEN \\ ${ }^{2}$ Materials and Manufacturing, Swerea SICOMP, S-94126 Piteå, SWEDEN
}

liva.rozite@1tu.se

It has been shown that natural fibers with high cellulose content (e.g. flax, hemp) compare well in terms of mechanical performance with glass fibers, especially if properties are normalized with density. However, wide utilization of these fibers in high performance composites is hindered by a number of shortcomings, such as poor adhesion between matrix and fibers, limited fiber length, difficulties associated with control of fiber alignment and orientation. Most probably one of the major drawbacks of natural fibers is variability of their properties (depending on location of growth and time of harvest). Therefore, another type of reinforcement with high cellulose content, continuous Regenerated Cellulose Fibers $(\mathrm{RCF})$, is of high interest. However mechanical behaviour of RCF is highly non-linear and as a result, performance of the composite with this reinforcement is also non-linear with presence of very significant visco-elastic behaviour.

The main objective of this investigation is to predict mechanical behaviour of these composites by developing existing models to capture their time-dependent behaviour. In order to identify and quantify parameters needed for the modelling, extensive damage tolerance tests as well as creep experiments are carried out. Preliminary results from tests of composite laminates with RCF show that damage in these materials are very similar to that of synthetic composites (like glass and carbon fiber composites). However, these results also show that stress-strain curves for these materials are very non-linear and loading-unloading experiments demonstrate that the shape of the stress-strain curve has the form of hysteresis loop. Therefore these materials have to be treated as highly non-linear with presence of visco-elastic and/or visco-plastic effects. 